\title{
The Key Methods to Increase the Success Rate of Endoscopic Repair of Cerebrospinal Fluid Rhinorrhea
}

\author{
Matin Ghazizadeh ${ }^{1}$ and Golfam Mehrparvar ${ }^{1, *}$ \\ ${ }^{1}$ Otorhinolaryngology, Head and Neck Surgery Department, Shahid Beheshti University of Medical Sciences, Tehran, Iran \\ "Corresponding author: Otorhinolaryngology, Head and neck Surgery Department, Shahid Beheshti University of Medical Sciences, Tehran, Iran. Tel: +98-9123499607, Email: \\ gmehrparvar@yahoo.com
}

Received 2018 October 27; Revised 2019 February 06; Accepted 2019 February 18.

\begin{abstract}
Objectives: To evaluate the success rate of endoscopic repair of CSF leak and factors with potential effect on surgical outcome.

Methods: A case series study based on review of medical records and follow up of patients admitted at three hospitals during a 5 year period.

Results: Of 43 operated patients 38 (88.4\%) had successful result after first surgical attempt. Defect size, number of graft layers, graft placement technique-underlay vs. overlay-lumbar drain placement or serial lumbar punctures did not show a statistically significant association with surgical outcome. Definite determination of defect site before or during operation had a relationship with surgical success with a P value of 0.06 there was a significant correlation between the number of layers on the defect site and immediate postoperative improvement.

Conclusions: Endoscopic repair of CSF leak has been proven as a successful method with reported success rate of above 80 percent. Careful attempt to find the exact site of CSF leak is recommended. We do not suggest the routine use of lumbar drains, serial post op lumbar punctures and intrathecal fluorescine due to their potential complications and no evidence for their efficacy.
\end{abstract}

Keywords: Cerebrospinal Fluid, Cerebrospinal Fluid Leak, Endoscopic Repair

\section{Background}

The central nervous system completely closed from the changeable context of blood by the blood-brain barrier and the blood-cerebrospinal fluid (CSF) barrier (1).

CSF is a clear, plasma-like fluid that bathes the central nervous system which has a physiologic volume of about $150 \mathrm{~mL}$. Normal CSF pressure is a function of a tightly maintained balance in rates of production and resorption of CSF.

CSF rhinorrhea results from a fistulous tract between the intracranial and nasal cavities. This fistula can be due to traumatic and non-traumatic causes. Although CSF leak most commonly occurs following trauma (80\% - 90\% of cases), other etiologies such as postoperative defect (10\%) and spontaneous leak (3\% - 4\%) should considered (2).

CSF fistulae persisting for $>7$ days has a significantly increased risk of developing meningitis. Therefore, surgical closure of leaks or defects at the skull base should be considered to prevent ascending meningitis. The goal of surgical therapy is repair of the dural defect contributing to the CSF leak (3).
The repair of CSF rhinorrhea has rapidly evolved over the past 30 years. Prior to the advent of the endoscopic approach, craniotomy was used for repairs.

Nowadays endoscopic repair is the standard method of treatment in most of the cases of CSF rhinorrhea. Since the development of these methods several attempts have been done to evaluate the factors influencing the results and different factors have been proposed as potential causes of surgical failure. Also many efforts have been undertaken to improve the outcome by manipulation of technical factors, using adjunctive methods or inventing new methods.

\section{Methods}

It was a cohort study with the aim of evaluation the success rate of endoscopic repair of CSF leak in first attempt and the overall success in patients admitted at three different medical university-affiliated hospitals during a 5 year period. We also assessed factors with potential effect on surgical outcome.

All patients with proved CSF rhinorrhea according to history, physical examination, endoscopic and paraclini- 
cal evaluations who were admitted for surgical repair included in this study.

Exclusion criterion was detection of defect in any area other than anterior skull base leading to CSF leakage.

The information of patients admitted in this time interval for surgical repair of CSF rhinorrhea was obtained from medical records. Follow up period varied from 2 to 29 months (mean: 9 months).

In order to assess patients' current status, history and physical examination including endoscopic evaluation were done.

In cases with evidences suggesting recurrent leak, appropriate paraclinical and imaging studies- beta 2 transferrin test, high resolution computed tomography or magnetic resonance imaging- were done and revision surgical repair was planned for patients with proved CSF leak.

Causes of leakage, associated symptoms and sites of defect were documented.

Variables with potential effect on surgical outcome (the material used for repair, number of graft layers, graft placement technique -underlay vs. overlay- lumbar drain placement or serial postop LPs) were identified in each patient.

$\chi^{2}$ test, $t$-test and Fisher exact test were used to determine statistical significance and also data analysis was undertaken by Stata software version 10.1.

\section{Results}

Forty-eight patients entered the study, 4 of them were excluded due to following reasons:

First of all, one patient with a history of previous failed endoscopic repair in which the second evaluation with intrathecal fluorescein showed the middle ear as the site of leakage.

Then, two cases of CSF leakage caused by dural involvement of skull base tumor with intracranial extension that underwent surgical repair using external approach.

Finally, one patient who died of meningitis before endoscopic surgery.

In analysis of demographic variables of 44cases remained in the study, normal distribution of age and gender variables were found. Patients were between 1 - 74 years of age (mean age of 35, SD: 18 ).

Among the studied patients 19 (43.2\%) were female and 25 (56.8\%) were male.

The time period passed after surgery ranged from 2 to 29 months (mean $=9$ months)

In the follow up period, 3 patients died of underlying disease not related to the surgery, all of them suffered from tumors with skull base involvement (however, none of them showed evidences of recurrent leak).
Additionally, median postoperative hospitalization time was 8.7 day (range 1 - 23 days).

And during the period of study, one of the cases was not available for follow up.

Of 43 operated patients 38 (88.4\%) had successful result after first surgical attempt and revision surgery was required for 5 (11.6\%) of whom 3 cases were operated (repeated endoscopic repair in 2 and external surgery in 1 patient), all of revision surgeries were successful.

Also 2 patients with failed first operation did not consent for revision surgery. So at the end, there were a total of 40 successful endoscopic repairs.

In 5 failed operation, the mean time until detection of recurrence was 68 days. The failed cases were as follows:

In one patient, repair had been done using only fat tissue.

In 2 cases the site was not found definitely during operation.

In one case the defect was on the lateral site of sphenoid sinus.

One patient had a foveal site of defect which was repaired with standard method using fat and fascial graft.

Table 1 shows etiologies of CSF leak in studied cases.

\begin{tabular}{lc}
\hline Table 1. Frequency of Different Etiologies of CSF Leak in the Studied Group \\
\hline Etiology & Frequency (\%) \\
\hline Trauma & $17(38.6)$ \\
\hline Iatrogenic (due to endoscopic sinus surgery) & $8(18.2)$ \\
\hline Iatrogenic (due to endoscopic pituitary surgery) & $4(9.1)$ \\
\hline Spontaneous & $8(18.2)$ \\
\hline Tumors & $5(11.4)$ \\
\hline Congenital anomalies & $2(4.5)$ \\
\hline Total & $44(100)$ \\
\hline
\end{tabular}

In evaluation of symptoms, all of the patients had presented with unilateral rhinorrhea.

Associated conditions are shown in Table 2.

It should be mentioned here that, the most common sites of defect were ethmoid roof, sphenoid sinus and cribriform plate respectively (Table 3 ).

Table 4 illustrates various approaches used for surgical repair of CSF leaks.

Table 5 demonstrates various graft materials applied in studied patients.

Graft placement techniques were not clearly mentioned in 11 cases, of 33 remained patients at least one layer of underlay graft had been used in 19 (43.2\%) and total overlay technique was found to be used in 14 (31.8\%).

Intra and post-operative lumbar drains were used in 5 


\begin{tabular}{|c|c|}
\hline Associated Conditions & Frequency (\%) \\
\hline Headache & $12(27.3)$ \\
\hline Meningitis & $9(20.5)$ \\
\hline Seizure & $6(13.6)$ \\
\hline Pneumocephalus & $4(9.1)$ \\
\hline Hyposmia & $4(4.5)$ \\
\hline \multicolumn{2}{|l|}{ Table 3. Frequency of Defect Sites } \\
\hline Defect Site & Frequency (\%) \\
\hline Ethmoid roof & $14(31.8)$ \\
\hline Sphenoid roof & $9(20.4)$ \\
\hline Cribriform plate & $8(18.2)$ \\
\hline Frontal sinus & $4(9.1)$ \\
\hline Lateral lamella & $2(4.55)$ \\
\hline Not clearly identified & $5(11.4)$ \\
\hline Not documented in records & $2(4.55)$ \\
\hline Total & $44(100)$ \\
\hline Approach & Frequency (\%) \\
\hline Trans ethmoidal & $30(68.2)$ \\
\hline Trans sphenoidal & $9(20.5)$ \\
\hline Trans nasal & $3(6.8)$ \\
\hline Frontal trephination & $2(4.5)$ \\
\hline Total & $44(100)$ \\
\hline
\end{tabular}

Table 5. Frequency of Different Materials Used for Surgical Repair of Skull Base Defect

\begin{tabular}{lc}
\hline Material & Frequency \\
\hline Mucosa & 24 \\
Muscle & 22 \\
\hline Fat & 15 \\
\hline Cartilage & 13 \\
\hline Fascia lata & 13 \\
\hline Temporal fascia & 13 \\
\hline Abdominal fascia & 2 \\
\hline Vascularized flap & 12 \\
\hline
\end{tabular}

of 44 patients. (11.4\%), serial postoperative lumbar puncture was used in 5 patients.

In order to determine factors affecting the success of endoscopic repair of CSF leak we did statistical analysis of following variables:

Defect size, number of graft layers, graft placement technique -underlay vs. overlay- lumbar drain placement or serial LPs were evaluated as potential effective factors, and none of them showed a statistically significant association with surgical outcome. Also, intrathecal fluorescein for detection of defect site, did not improve the results.

Review of operative notes exhibited that in more than half of the patients at least one layer of mucosal or fascial graft had been used, and muscle, fat and cartilage were in lower orders of frequency.

By using fisher exact test, no association was found between surgical outcome and history of previous surgery endoscopic or external- or type of endoscopic approach.

Based on the fisher exact test, definite determination of defect site before or during operation had a relationship with surgical success with a P value of 0.06 .

Immediate postoperative improvement of leakage was observed in 31 patients (70.5\%), postoperative status in immediate postop period was not mentioned in one case (2.3\%) and in 12 cases (27.3\%) leak did not stop instantly.

We also evaluated factors influencing immediate postop improvement, etiology of leak, size and site of defect, history of previous surgical treatment, duration of leak, the applied surgical approach, the material used, and graft placement technique did not affect the outcome.

The mean numbers of layers used for defect repair was 2.5 in total studied group, average number of graft layers applied for repair of defect was 3 in 31 patients in whom CSF leak stopped promptly after the operation and it was 2 for other 12.

The $t$-test was utilized to analyze the immediate postsurgical status according to the number of graft or flap layers used for defect repair which showed a statistically significant association (P value: 0.046).

There was also a significant correlation between the number of layers on the defect site and immediate postoperative improvement based on the Mann-Whitney test (Wilcoxon W) (P value: 0.037).

Also, $\chi^{2}$ test showed a statistically significant relation between the use of muscle tissue as graft material and immediate cessation of leak (P value: 0.03).

In addition, multifactorial logistic regression analysis showed that the relation of muscular graft and immediate leak cessation was not a dependent one, in other words patients with more layers of graft had more muscular tissues as well.

\section{Discussion}

High success and low complication rate of endoscopic approach reported in various trials has made it the stan- 
dard treatment method of anterior cranial base defects in many situations (4-11).

A recent literature review study on the success rate of trans nasal endoscopic repair of CSF rhinorrhea showed a mean success rate of 90\% (range: 60\% -100\%) (4).

Comparable results were obtained in our study population with $88.4 \%$ improvement in first attempt and $100 \%$ success rate of revision surgeries.

In our population of study the most common causes of leak were found to be trauma (38.6\%) and iatrogenic (27.3\%) in order of frequency, the primary iatrogenic cause was endoscopic sinus surgery which is somehow similar to previous studies reviewed by Daele et al. in 2011 (12).

The most common site of skull base defect in our patients as a whole was ethmoid roof similar to previous reports $(6,8)$ though differentiating the patients according to etiology of leak, revealed the frontal and sphenoid region as the most prevalent site in post-traumatic leaks and cribriform plate and anterior ethmoid area in spontaneous leaks. This is different from previous studies to some extent in which accidental injuries were reported to involve cribriform plate mostly and spontaneous leaks were due to a defect of lateral rescess of sphenoid sinus in most of the cases $(13,14)$.

Current study showed that neither the graft or flap type nor the graft placement technique -underlay vs. overlay- affect the outcome.

The accurate determination of skull base defect was the variable with more correlation with surgical success in our study, although not statistically significant (P value: 0.06 ) it may be significant in a larger sample size.

The clinical parameters affecting endoscopic repair of CSF leaks has been the subject of different studies conducted in various designs to identify factors regarding the patient, CSF fistula, and treatment that may influence the results of the repair.

Lee et al. in their study of 28 patients found that among the variables affecting initial endoscopic success, the location of CSF leak and direct visualization were significant factors (11).

Hegazy et al. in a meta-analysis of all published studies in a 9-year period, reported that the success rate of repairs using any of the reported techniques -overlay and underlay- and materials -vascularized flap, mucosa, allograft, fascia lata, temporalis fascia and fat grafts- were high and not statistically different (5).

Using intrathecal fluorescein during the surgery did not affect the outcome, maybe due to absence of active flow in patients with undetermined defect site in whom intrathecal fluorescein was used, which lead to inefficacy of this technique for precise detection of leak site. So we do not recommend routine use of intrathecal fluorescein.
In an attempt to increase success rates, lumbar drain placement during or after surgery or serial postop LP s, has been proposed as an adjunctive measure but with conflicting results.

Lobo et al, in a systemic review on articles from January 1, 2000 until June 30, 2016 found that in most cases of spontaneous leak, intraoperative placement of lumbar drain did not appear to result in improved success rates for either anterior or lateral skull base leaks (15).

Similarly Albu et al. in a randomized prospective study assessed the relationship between use of LDs and recurrent leaks and resulted that success rates of CSF repair were not associated with the application of LDs (16). A large series by Oles et al. published their 10 years' experience on endoscopic closure of CSF leaks (36 patients) and demonstrated high success rate (overall success rate of $97.2 \%$ ) which had been achieved without the use of lumbar drain (17).

Our study population also did not show any significant benefit for this intervention.

Since the observation of successful cessation of CSF leakage in the immediate postoperative period in group patients, we looked at factors that may influence this outcome.

Among factors evaluated, number of layers utilized for repair related to the result. Average layers used was 3 in cases with instant leak improvement and 2 in the other patients, but the graft material and grafting technique were not significant factors. As we know, no previous study has evaluated this outcome and related factors.

Therefore we suggest using at least 3 layers of graft/flap in the defect site.

\subsection{Conclusions}

Endoscopic repair of CSF leak has been proven as aprosperous method with reported success rate of above 80 percent as described in different reports.

Among different variables with potential effect on outcome, we found more significant association with determination of defect site before or during surgery.

We recommend careful attempt to find the exact site of CSF leak and applying at least 3 layers of materials in defect site.

Routine use of lumbar drains, serial post op LP s and intrathecal fluorescein are not recommended considering their potential complications and also there is no evidence for their efficacy.

\section{Footnotes}

Authors' Contribution: Study concept, design and acquisition of data: Matin Ghazizadeh; analysis and interpretation of data and statistical analysis: Matin Ghazizadeh and 
Golfam Mehrparvar; drafting of the manuscript and critical revision of the manuscript: Golfam Mehrparvar.

Conflict of Interests: There is no financial interests related to the material in the manuscript.

Ethical Approval: The study has been approved by Ethical Committee of Tehran University of Medical Sciences.

Funding/Support: It is not declared by the authors.

\section{References}

1. Engelhardt B, Sorokin L. The blood-brain and the blood-cerebrospinal fluid barriers: Function and dysfunction. Semin Immunopathol. 2009;31(4):497-511. doi: 10.1007/s00281-009-0177-0. [PubMed: 19779720].

2. Adigun OO, Al-Dhahir MA. Anatomy, head and neck, cerebrospinal fluid. 2019

3. Abuabara A. Cerebrospinal fluid rhinorrhoea: Diagnosis and management. Med Oral Patol Oral Cir Bucal. 2007;12(5):E397-400. [PubMed: 17767107].

4. Sharma SD, Kumar G, Bal J, Eweiss A. Endoscopic repair of cerebrospinal fluid rhinorrhoea. Eur Ann Otorhinolaryngol Head Neck Dis. 2016;133(3):187-90. doi: 10.1016/j.anorl.2015.05.010. [PubMed 26776882].

5. Hegazy HM, Carrau RL, Snyderman CH, Kassam A, Zweig J. Transnasal endoscopic repair of cerebrospinal fluid rhinorrhea: A metaanalysis. Laryngoscope. 2000;110(7):1166-72. doi: 10.1097/00005537200007000-00019. [PubMed:10892690].

6. Alexander A, Mathew J, Varghese AM, Ganesan S. Endoscopic repair of CSF fistulae: A ten year experience. J Clin Diagn Res. 2016;10(8):MC014. doi: 10.7860/JCDR/2016/18903.8390. [PubMed: 27656471]. [PubMed Central: PMC5028510].

7. Mishra SK, Mathew GA, Paul RR, Asif SK, John M, Varghese AM, et al. Endoscopic repair of CSF rhinorrhea: An institutional experience. Iran
J Otorhinolaryngol. 2016;28(84):39-43. [PubMed: 26878002]. [PubMed Central: PMC4735615].

8. Castelnuovo P, Mauri S, Locatelli D, Emanuelli E, Delu G, Giulio GD. Endoscopic repair of cerebrospinal fluid rhinorrhea: Learning from our failures. Am J Rhinol. 2001;15(5):333-42. [PubMed: 11732821].

9. Mirza S, Thaper A, McClelland L, Jones NS. Sinonasal cerebrospinal fluid leaks: Management of 97 patients over 10 years. Laryngoscope. 2005;115(10):1774-7. doi: 10.1097/01.mlg.0000175679.68452.75. [PubMed: 16222193].

10. McMains KC, Gross CW, Kountakis SE. Endoscopic management of cerebrospinal fluid rhinorrhea. Laryngoscope. 2004;114(10):1833-7. doi: 10.1097/00005537-200410000-00029. [PubMed: 15454781].

11. Lee DH, Lim SC, Joo YE. Treatment outcomes of endoscopic repairs of sinonasal cerebrospinal fluid leaks. J Craniofac Surg. 2011;22(4):126670. doi: 10.1097/SCS.0b013e31821c6ad3. [PubMed: 21772201]

12. Daele JJ, Goffart Y, Machiels S. Traumatic, iatrogenic, and spontaneous cerebrospinal fluid (CSF) leak: Endoscopic repair. B-ENT. 2011;7 Suppl 17:47-60. [PubMed: 22338375]

13. Kerr JT, Chu FW, Bayles SW. Cerebrospinal fluid rhinorrhea: diagnosis and management. Otolaryngol Clin North Am. 2005;38(4):597-611. doi: 10.1016/j.otc.2005.03.011. [PubMed: 16005720].

14. Schlosser RJ, Wilensky EM, Grady MS, Bolger WE. Elevated intracranial pressures in spontaneous cerebrospinal fluid leaks. Am J Rhinol. 2018;17(4):191-5. doi: 10.1177/194589240301700403.

15. Lobo BC, Baumanis MM, Nelson RF. Surgical repair of spontaneous cerebrospinal fluid (CSF) leaks: A systematic review. Laryngoscope Investig Otolaryngol. 2017;2(5):215-24. doi: 10.1002/lio2.75. [PubMed: 29094066]. [PubMed Central: PMC5655559].

16. Albu S, Emanuelli E, Trombitas V, Florian IS. Effectiveness of lumbar drains on recurrence rates in endoscopic surgery of cerebrospinal fluid leaks. Am J Rhinol Allergy. 2013;27(6):e190-4. doi: 10.2500/ajra.2013.27.3986. [PubMed: 24274213].

17. Oles K, Skladzien J, Leszczynska J. Transnasal endoscopic treatment of cerebrospinal fluid leaks: 10 years' experience. B-ENT. 2013;9(3):201-6. [PubMed: 24273951]. 